\title{
Emigración de profesionales de enfermería en México
}

Emigration of nursing professionals in Mexico

Emigração de profissionais de enfermagem no México

Como citar este artículo: Fernández-Sánchez Higinio, Enríquez-Hernández Claudia Beatriz, Zapíen Vázquez María de los Ángeles, Horcasitas-Tovar Aimée Giselle. Emigración de profesionales de enfermería en México. Revista Cuidarte. 2021;12(1):e2008. http://dx.doi.org/10.15649/cuidarte.2008

Revista Cuidarte

doi. Rev Cuid. Ene. - Abril. 2021; 12(1): e2008 http://dx.doi.org/10.15649/cuidarte.2008

E-ISSN: 2346-3414

(1) Higinio Fernández-Sánchez ${ }^{1}$

(1) Claudia Beatriz EnríquezHernández ${ }^{2}$

(1) María de los Ángeles Zapíen Vázquez ${ }^{3}$

(1) Aimée Giselle Horcasitas-Tovar ${ }^{4}$

1 University of Alberta, Faculty of Nursing, Edmonton Clinic Health Academy. Autor de correspondencia E-mail: higinio@ualberta.ca

2 Federación Mexicana de Asociaciones de Facultades y Escuelas de Enfermería A.C. (FEMAFEE), E-mail: beenriquez@uv.mx

3 Red Mexicana de Enfermería en la Prescripción A.C. E-mail: redprescripcionenf@gmail.com

4 Asociación Mexicana de Estudiantes de Enfermería A. C. (AMEENF). E-mail: aimeehorcasitas@gmail.com

Estimada editora,

La creciente emigración de profesionales de enfermería en México podría colocar su sistema de salud en una crisis nacional. Actualmente, existen más de 272,000 migrantes a nivel global'. Se estima que $11,800,000$ son mexicanos, el $35 \%$ de ellos son profesionistas ${ }^{2}$. México se ubica en sexto lugar de países que envían migrantes calificados y en primer lugar en la región de América Latina. Los principales países receptores son Estados Unidos (EE. UU.), Canadá, y España. En 2015, en Estados Unidos el número de mexicanos calificados rebasó las 250,000. De acuerdo con datos de la Organización Mundial de la Salud ${ }^{3}$ hay más de 20,700,000 de profesionales de enfermería y partería en el mundo. No obstante, se calcula que para el 2030 nos enfrentaremos ante la escases de 18,000,000. Tan solo en Canadá este déficit podría llegar a los 60,000 enfermeros, mientras que en EE. UU esta cifra se triplica a los 240,000 .

Los gobiernos de países desarrollados continúan implementando estrategias para atraer y retener talentos internacionales que contribuyan al desarrollo de sus economías. En este sentido, se han puesto en marcha procesos migratorios que facilitan la entrada de profesionistas de otros países. Por ejemplo, Canadá tiene el programa Express Entry, que es un sistema basado en puntos que toma en cuenta varios factores, entre otros, grados académicos, edad, e idiomas. Así mismo, desde hace varios años EE. UU. otorga las visas H-1B que están destinadas a personas con titulo de enseñanza superior o su equivalente.

Recibido: 27 de noviembre de 2020 Aceptado: 17 de diciembre de 2020 Publicado: 19 de febrero de 2021 $\square *$ Correspondencia Higinio Fernández-Sánchez E-mail: higinio@ualberta.ca 
Respecto a la enfermería, la evidencia empírica señala que más de 17,000 enfermeros mexicanos ejercían en EE. UU. entre 2009 y $2011^{4}$. Aunado al déficit de enfermeros a nivel mundial, los enfermeros mexicanos podrían tener otros motivos para emigrar. Mientras que en México los licenciados en enfermería perciben un promedio de 323,186MXN anuales, en EE. UU. llegan a divisar más de 79,696USD 5 . Además, la falta de reconocimiento de sus grados académicos, la sobrecarga de trabajo, las condiciones laborales precarias, la pobre remuneración económica, la insatisfacción laboral, y la inseguridad del país también podrían ser factores que orillan a los enfermeros a emigrar.

Enfermería representa casi el $50 \%$ de la fuerza laboral de salud, lo cual es relevante en estos momentos de crisis sanitaria mundial por la COVID-193. Se ha puesto de manifiesto el reconocimiento al trabajo y la fortaleza de los profesionales de enfermería para enfrentar esta pandemia, resaltando la importancia para la salud de la población en México y en el mundo. Sin embargo, no basta con un reconocimiento público por parte de diversos organismos y gobiernos, debe también verse reflejado en las mejoras de las condiciones de trabajo, repercutiendo en la mejora de la calidad de vida de los profesionales de enfermería. Todo esto contribuye para consolidar el reconocimiento social de la profesión.

Ante este panorama, exhortamos al gobierno de México a que implementen acciones para la retención de estos recursos humanos al extranjero. Entre otras, sugerimos que México: sugerimos que México: Facilite la participación de enfermería en las políticas públicas de salud.

- Invierta en la formación de recursos humanos de enfermería de alta calidad.

- $\quad$ Asegure la evaluación permanente de escuelas y facultades de enfermería para alcanzar los estándares de calidad y acreditación.

- Mejore las condiciones laborales.

- $\quad$ Reconozca en todo el país el nivel de licenciatura y de posgrado al momento de la contratación.

- Unifique las categorías de contratación de nivel profesional en las diversas instituciones de salud públicas y privadas.

- $\quad$ Aumente el salario.

- $\quad$ Promueva la emancipación de género.

- $\quad$ Regule y promueva la práctica avanzada de enfermería.

- $\quad$ Facilite la participación de enfermería en las políticas públicas de salud.

- Reclutamiento de profesionales de enfermería para puestos de toma de decisiones.

- $\quad$ Apoye la investigación de enfermería y reconozca a aquellos que la realizan.

- $\quad$ Pondere la prevención de factores psicosociales y riesgos laborales en enfermería.

- $\quad$ Fomente el liderazgo enfermero, especialmente a las enfermeras mujeres.

- Reconozca y dignifique a los profesionales enfermeros.

- Impulse la educación continua.

- $\quad$ Proporcione las herramientas suficientes para llevar a cabo el cuidado de manera adecuada. (hablando de recursos físicos como material, instalaciones, etc.) 
En México, como muchos otros países en el mundo se observan las consecuencias de esta escases de recursos humanos enfermeros, de no tomar cartas en el asunto la emigración de estos profesionistas podría empeorar su situación en los próximos años.

Conflicto de interés: Los autores no declaran tener conflicto de interés.

\section{Referencias}

1. Naciones Unidas. Migration [internet]. 2020. [citado 11 de noviembre de 2020]. Disponible en: https://www.un.org/en/sections/issues-depth/migration/index.html

2. Secretaría de Relaciones Exteriores. Población mexicana en el mundo [internet]. 2017. [citado 11 de noviembre de 2020]. Disponible en:

http://www.ime.gob.mx/estadisticas/mundo/estadistica_poblacion_pruebas.html

3. World Health Organization. Nursing and Midwifery- WHO Global Strategic directions for strenghtening Nusring and midwifery 2016-2020 [internet]. 2020. [citado 11 de noviembre de 2020]. Disponible en: https://www.who.int/hrh/nursing_midwifery/nursing-midwifery/en/

4. Hualde-Alfaro A, Rosales-Martínez Y. Profesionales que emigran. Una comparación entre enfermeras e ingenieros mexicanos en Estados Unidos. Espiral Estudios sobre Estado y Sociedad. 2017;24(70):181-219. https://doi.org/10.32870/espiral.v24i70.6119

5. Economic Research Institute. Registered Nurse Salary [internet]. 2020. [citado 11 de noviembre de 2020]. Disponible en:

https://www.erieri.com/salary/job/registered-nurse/united-states 
